\title{
Campylobacter jejuni response to human mucin MUC2: modulation of colonization and pathogenicity determinants
}

\begin{abstract}
Correspondence
George L. Mendz

GMendz@nd.edu.au
\end{abstract}

Received 6 November 2007

Accepted 3 February 2008

\section{Quoc V. Tu, ${ }^{1}$ Michael A. McGuckin ${ }^{2}$ and George L. Mendz ${ }^{1,3}$}

\author{
${ }^{1}$ School of Medical Sciences, The University of New South Wales, Sydney, NSW 2052, Australia \\ ${ }^{2}$ Mucosal Diseases Program, Mater Medical Research Institute, Mater Misericordiae Hospitals, \\ South Brisbane, OLD 4101, Australia \\ ${ }^{3}$ School of Medicine, Sydney, The University of Notre Dame Australia, Darlinghurst, NSW 2010, \\ Australia
}

\begin{abstract}
Campylobacter jejuni is the main cause of bacterial acute gastroenteritis worldwide. In its colonization of the host intestinal tract, it encounters secreted mucins in the mucus layer and surface mucins in the epithelial cells. Mucins are complex glycoproteins that comprise the major component of mucus and give mucus its viscous consistency. MUC2 is the most abundant secreted mucin in the human intestine; it is a major chemoattractant for C. jejuni, and the bacterium binds to it. There are no studies on the transcriptional response of the bacterium to this mucin. Here, cell-culture techniques and quantitative RT-PCR were used to characterize in vitro the effects of MUC2 on C. jejuni growth and the changes in expression of $20 \mathrm{C}$. jejuni genes related to various functions. The genes encoding cytolethal distending toxin protein ( $c d t A B C$ ), vacuolating cytotoxin $(\operatorname{vac} B), C$. jejuni lipoprotein (j/pA), Campylobacter invasion antigen (ciaB), the multidrug efflux system ( $c m e A B$ ), putative mucin-degrading enzymes (cj1344c, cj0843c, cj0256 and cj1055c), flagellin A ( $f l a A)$ and putative rod-shape-determining proteins (mreB and $m r e C$ ) were upregulated, whereas those encoding Campylobacter adhesion fibronectin-binding protein (cadF) and sialic acid synthase (neuB1) were downregulated. These results showed that C. jejuni utilizes MUC2 as an environmental cue for the modulation of expression of genes with various functions including colonization and pathogenicity.
\end{abstract}

\section{INTRODUCTION}

Campylobacter jejuni is a Gram-negative, spiral, motile bacterium that colonizes the intestine of vertebrates. The bacterium is the main cause of human acute bacterial gastroenteritis in both developing and developed countries (Allos, 2001). Common symptoms of human C. jejuni infection are diarrhoea, fever, vomiting and abdominal pain. C. jejuni infection is a leading cause of childhood morbidity and mortality in the developing world (Allos, 2001). It is associated with the development of intestinal mucosa-associated lymphoid tissue lymphoma (Lecuit et al., 2004) and with post-infection autoimmune diseases such as Guillain-Barré and Miller Fisher syndromes (Yuki \& Koga, 2006). Its various pathogenic effects indicate that C. jejuni infection poses a significant global public health problem.

The mucosal tissues of the gastrointestinal, respiratory, urinary and reproductive tracts, as well as the surfaces of several organs such as the eye, nose and mouth, are

Abbreviation: PAS, periodic acid-Schiff reagent. exposed to the external environment. The mucus layer present in these tissues provides protection to underlying mucosal epithelial cells against chemical, enzymic, microbial and mechanical insult (Neutra \& Forstner, 1987; Strugala et al., 2003). The major components of the mucus layer are secreted gel-forming mucins, complex glycoproteins that give mucus its viscous consistency.

Most pathogenic bacteria that colonize the gastrointestinal tract subvert the mucus barrier by effective motility through the gel and the use of enzymes capable of degrading mucin carbohydrates and/or mucins. MUC2 is the most common gel-forming mucin secreted by goblet cells of the small intestine and colon. The interactions of bacteria with this mucin elicit responses in them that are not well characterized at the molecular level and are not restricted to the modulation of the expression of enzymes involved in mucus modification. Mucins are major chemoattractants for C. jejuni (Hugdahl et al., 1988) and the bacterium binds to them (McAuley et al., 2007; Szymanski et al., 1995), indicating that mucins carry the physiologically relevant oligosaccharide ligands for the 
adhesins of this bacterium. A very small number of molecular studies have been conducted to investigate the interactions between C. jejuni and human MUC2, but they used poorly characterized bovine mucin preparations (Hugdahl et al., 1988). Knowledge of the responses of $C$. jejuni to the mucus layer will be another step in understanding the mechanisms of $C$. jejuni infection. The aim of this work was to determine the effects of MUC2 on C. jejuni growth and to characterize the changes induced by this mucin in the expression of 20 bacterial genes related to virulence, putative mucin-degrading enzymes, cell morphology, adhesion and motility.

\section{METHODS}

Bacterial culture techniques. C. jejuni strain NCTC 11168 was employed in this work as this strain was isolated from a human patient and its genome has been sequenced. Strain NCTC 11168 was cultured on Campylobacter selective agar (CSA) medium at $37{ }^{\circ} \mathrm{C}$ under the microaerobic conditions of $5 \% \mathrm{O}_{2}, 5 \% \mathrm{CO}_{2}$ and $90 \% \mathrm{~N}_{2}$ in a Sanyo $\mathrm{O}_{2} / \mathrm{CO}_{2}$ Tri-gas incubator (Quantum Scientific). CSA medium comprises blood agar base 2 (Oxoid) supplemented with $6 \%$ defibrinated horse blood (Oxoid), $0.32 \mu \mathrm{g}$ polymyxin $\mathrm{ml}^{-1}, 5 \mu \mathrm{g}$ trimethoprim $\mathrm{ml}^{-1}, 10 \mu \mathrm{g}$ vancomycin $\mathrm{ml}^{-1}$ and $2 \mu \mathrm{g}$ Fungizone (Bristol-Myers Squibb) $\mathrm{ml}^{-1}$. Culture purity was verified using phasecontrast microscopy, Gram-staining and tests for catalase and oxidase activities by adding hydrogen peroxide and tetramethyl- $p$-phenylenediamine, respectively, to cell suspensions.

Purification and characterization of human mucin MUC2. Mucus was purified from urine samples obtained from patients with artificial bladders made of intestinal tissue that secretes mucins abundantly (Gerharz et al., 2003; Nabi et al., 2005). Samples were obtained from patients in the first few days following surgery to avoid any change in mucin glycosylation that may occur in response to epithelial changes in the urinary tract microenvironment. The extraction of MUC2 was performed based on a published protocol (Davies \& Carlstedt, 2000) with some modifications. Briefly, mucus collected from urine was subjected to repetitive extractions in $6 \mathrm{M}$ guanidinium chloride, $5 \mathrm{mM}$ EDTA and $10 \mathrm{mM}$ sodium phosphate $(\mathrm{pH} 7.0)$. The insoluble fraction containing oligomerized MUC2 depleted of contaminating non-covalently attached proteins was reduced in $10 \mathrm{mM} \mathrm{DTT}, 0.1 \mathrm{M}$ Tris/ $\mathrm{HCl}(\mathrm{pH} \mathrm{8.0)}$ for $24 \mathrm{~h}$ and alkylated in $25 \mathrm{mM}$ iodoacetamide at $4{ }^{\circ} \mathrm{C}$ for $24 \mathrm{~h}$. Reduction of disulfide bonds disassembles the mucin homo-oligomers, thereby greatly reducing viscosity and solubilizing the mucins. Reduced, purified MUC2 was dialysed against water before addition to bacterial cultures. Mucin purity was verified by $1 \%$ agarose gel electrophoresis for $8 \mathrm{~h}$, transfer onto nitrocellulose membranes and staining with periodic acid-Schiff reagent (PAS) and Western blotting with MUC2 rabbit antiserum primary antibody (LUM 2-3; a kind gift of Professor I. Carlstedt, Lund University, Sweden). PAS identifies mucin oligosaccharides, and a single PAS-positive band was obtained, which co-localized precisely with the results of MUC2 Western blotting, verifying that MUC2 was the mucin present in these preparations. Purified mucin was also subjected to standard SDS-PAGE and staining with Coomassie blue, which revealed only negligible contamination with non-mucin proteins that co-purified with MUC2.

Growth inhibition studies. The growth response of $C$. jejuni as a function of MUC2 concentration was determined. Frozen cultures were thawed to room temperature and $20 \mu \mathrm{l}$ bacterial suspension was spread on CSA plates and incubated at $37^{\circ} \mathrm{C}$ under microaerobic conditions. After $24 \mathrm{~h}$ incubation, bacteria were harvested in $2 \mathrm{ml}$ brain heart infusion (BHI) medium, transferred to $10 \mathrm{ml} \mathrm{BHI}$ medium in $25 \mathrm{ml}$ vented flasks with $0.2 \mu \mathrm{m}$ filter caps and grown for $12 \mathrm{~h}$ under the same conditions. Bacteria were harvested by centrifuging the cultures for $5 \mathrm{~min}$ at $5000 \mathrm{~g}$ at room temperature. The pellets were resuspended in $50 \mathrm{ml}$ fresh sterile BHI medium and the suspension was adjusted to an $\mathrm{OD}_{600}$ of 0.5 . Three millilitres of bacterial suspension was dispensed into each well of Cellstar six-well plates (Greiner Bio One) and cultures were supplemented with MUC2 at concentrations of between 0.01 and $2 \mathrm{mg} \mathrm{ml}^{-1}$. The growth rates of C. jejuni were determined spectrophotometrically by measuring the $\mathrm{OD}_{600}$ of cell suspensions and by colony counts (c.f.u. $\mathrm{ml}^{-1}$ ) at 0 and $12 \mathrm{~h}$ incubation. Thirty microlitres of serial dilutions of $10^{-2}$ to $10^{-9}$ were plated on CSA plates and incubated microaerobically, and the number of colonies was counted in triplicate for each dilution after incubation for 1 day under microaerobic conditions. Growth rates were measured for each MUC2 concentration by the difference in growth at both time points in three independent cultures $(n=3)$.

To compare the growth characteristics of $C$. jejuni in the presence or absence of MUC2, growth curves of the bacterium were measured at a mucin concentration that caused a measurable decrease in bacterial growth but was not lethal to the cells. Bacterial suspensions were prepared and dispensed in six-well plates, and cultures were

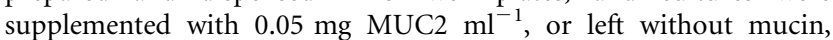
and incubated microaerobically. At $0,3,6,9,12,15,18$ and $24 \mathrm{~h}$, the densities of viable bacteria were determined in duplicate by counting c.f.u. $\mathrm{ml}^{-1}$ of serial dilutions of the cultures. The experiment was repeated twice with independent cultures $(n=2)$.

Sampling and total RNA extraction from C. jejuni liquid cultures. C. jejuni was grown microaerobically in liquid cultures at $37{ }^{\circ} \mathrm{C}$ in shaking vented flasks containing $10 \mathrm{ml}$ BHI medium for $12 \mathrm{~h}$. The cell suspensions were centrifuged at room temperature for $5 \mathrm{~min}$ at $5000 \mathrm{~g}$ and the pellets were resuspended in $10 \mathrm{ml} \mathrm{BHI}$ medium to an initial $\mathrm{OD}_{600}$ of 0.3 . MUC2 was added to the cultures at a concentration of $0.05 \mathrm{mg} \mathrm{ml}^{-1}$. Cultures with or without MUC2 were incubated microaerobically at $37^{\circ} \mathrm{C}$ and harvested at $12 \mathrm{~h}$. For total RNA isolation, $10 \mathrm{ml}$ bacterial suspension was mixed with $2 \mathrm{ml}$ RNAprotect Bacterial Reagent (Qiagen). Bacterial cells were pelleted by low-speed centrifugation for $10 \mathrm{~min}$ at $5000 \mathrm{~g}$ at room temperature. The pellets were resuspended in $200 \mu \mathrm{l}$ DEPC-treated lysozyme in TE buffer consisting of $30 \mathrm{mg}$ chicken lysozyme $\mathrm{ml}^{-1}$ ( $50000 \mathrm{U} \mathrm{mg}^{-1}$; Sigma-Aldrich), $10 \mathrm{mM}$ Tris/HCl (pH 8.0), $1 \mathrm{mM}$ EDTA and $10 \mu \mathrm{l}$ proteinase $\mathrm{K}\left(600 \mathrm{mAU} \mathrm{ml}^{-1}\right.$; Qiagen). Total RNA was extracted using Qiagen RNeasy mini columns and DNase treatment to remove traces of genomic DNA, following the manufacturer's instructions. Total RNA samples were resuspended in $20 \mu \mathrm{l}$ RNase-free DEPC-treated water; $6 \mu \mathrm{l}$ total RNA was set aside for the immediate assessment of RNA quantity and quality, and the rest was stored at $-80{ }^{\circ} \mathrm{C}$ for further experiments.

RNA purity and the presence of genomic DNA were assessed following the protocol published in Qiagen's RNeasy Handbook. Using $2 \mu \mathrm{l}$ aliquots of the samples, the RNA quality and quantity were also measured with an ND-1000 NanoDrop UV-Vis spectrophotometer (NanoDrop Technologies). Values of $A_{260} / A_{280}>2.0$ and $A_{260} / A_{230}>2.0$ are indicative of no protein and solvent contamination, respectively.

Reverse transcription: cDNA synthesis. Total RNA was reversetranscribed using a SuperScript III First-Strand Synthesis System for quantitative RT-PCR according to the manufacturer's instructions (Invitrogen). The purity and quantity of cDNA were assessed using an ND-1000 NanoDrop UV-Vis spectrophotometer as above.

Quantitative real-time RT-PCR. Primers were designed based on the published C. jejuni NCTC 11168 genome sequence (GenBank 
Table 1. Forward and reverse primer sets for quantitative real-time PCR

The primer set includes those for target genes and for the 16S rRNA gene as an internal control.

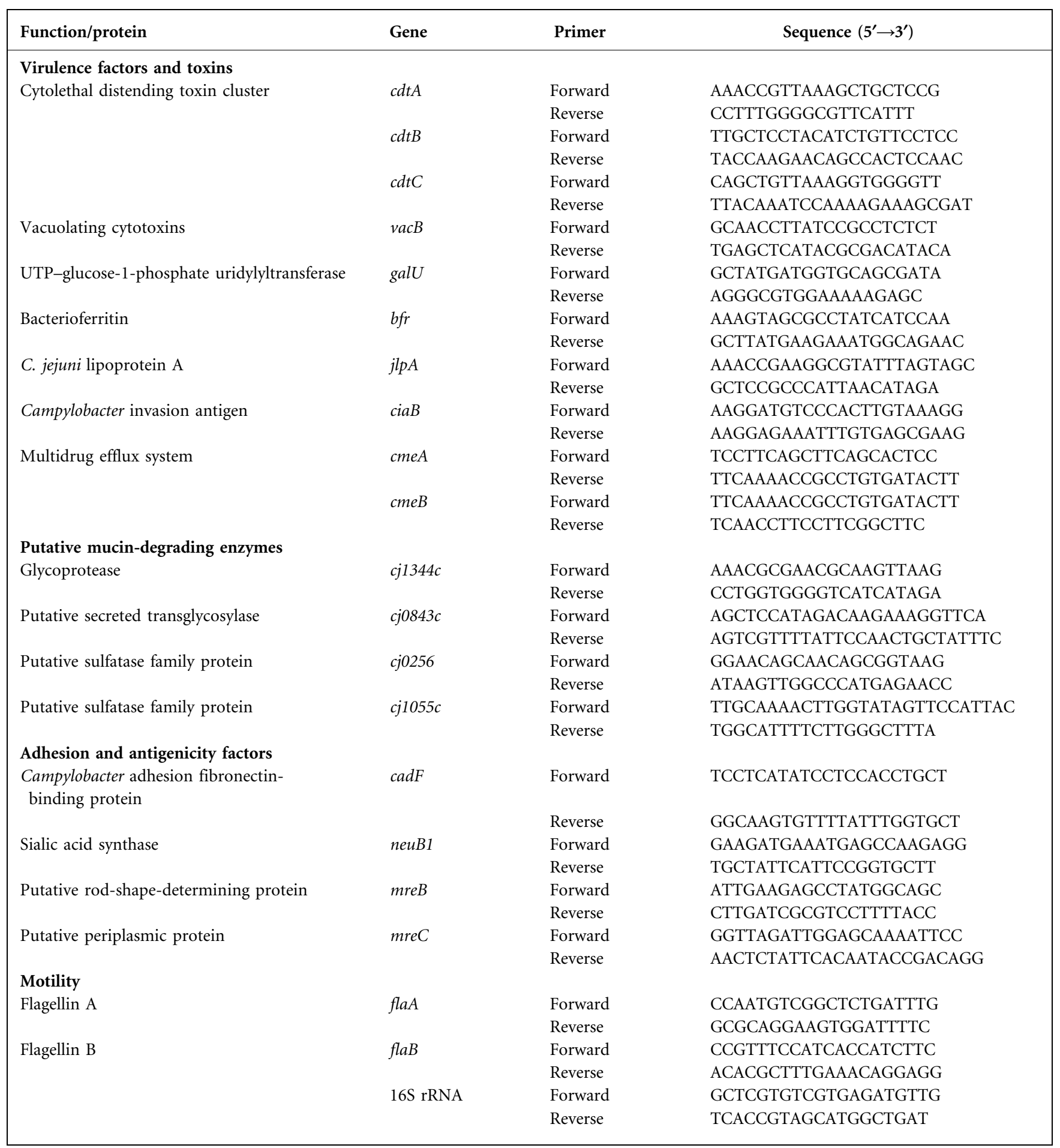

accession no. AL111168; Parkhill et al., 2000) using Primer3 (Rozen \& Skaletsky, 1998). The sequences of the primers used in this study are listed in Table 1. They all had the following properties: melting temperatures of between 61 and $63{ }^{\circ} \mathrm{C}$, length $19-23 \mathrm{nt}$, GC content 50-80 mol\% and expected product size of 300-310 bp. BLAST searches (Altschul et al., 1997) were performed against other bacterial genomes to determine the specificity of the primers.
Quantitative RT-PCR was performed using SYBR GreenER qPCR Supermix Universal following the manufacturer's protocol (Invitrogen) with a Rotor-Gene 3000 thermal cycler (Corbett Life Sciences) using 72-strip tubes. The concentration of cDNA in each sample was adjusted to $200 \mathrm{ng} \mu^{-1}$ using DEPC-treated water. PCRs were carried out under low-intensity light conditions and contained $12.5 \mu \mathrm{l}$ Supermix, $1.0 \mu \mathrm{l}$ forward primer $\left(100 \mathrm{ng} \mathrm{ll}^{-1}\right), 1.0 \mu \mathrm{l}$ reverse 
primer $\left(100 \mathrm{ng}^{-1} \mathrm{l}^{-1}\right), 1.0 \mu \mathrm{l} \mathrm{cDNA}$ and DEPC-treated water to a total volume of $25 \mu \mathrm{l}$. Each cDNA sample was amplified in technical triplicates for three different biological samples $(n=3)$. To check whether the primers amplified only the target and gave accurate quantification of the results, non-template controls for each gene were included in each run to make sure that no primer dimers were present after 40 thermal cycles. In addition, the melting curves of PCR products were used to assess the presence of primer dimers, contamination with genomic DNA and non-specific homologous sequences.

The relative expression ratio of genes was calculated based on the $C_{\mathrm{t}}$ comparative threshold cycle method (Livak \& Schmittgen, 2001). Primer efficiencies in this study were assumed to be 2 , as they were between 85 and $90 \%$ (Livak \& Schmittgen, 2001). Statistical analyses were performed on the ratio $R$ using the pair-wise randomization test of the REST software (Pfaffl et al., 2002). Genes were considered to be significantly regulated when the gene expression normalized to the $16 \mathrm{~S}$ rRNA reference gene differed significantly from the control $(P<0.05)$.

\section{RESULTS AND DISCUSSION}

\section{Inhibition of C. jejuni growth in the presence of MUC2}

The growth of C. jejuni NCTC 11168 in cultures supplemented with MUC2 at concentrations of 0.01$2.00 \mathrm{mg} \mathrm{ml}^{-1}$ was compared with growth of the bacterium in cultures with no MUC2. Table 2 shows the changes in bacterial growth given as the number of c.f.u. $\mathrm{ml}^{-1}$ for five different concentrations of MUC2; growth inhibition was measured at mucin concentrations from 0.05 to $2.00 \mathrm{mg}$ $\mathrm{ml}^{-1}$ without becoming lethal to the bacterium (Table 2). There were no significant changes in bacterial morphology as determined by light microscopy. Under the experimental conditions used, this decrease in $C$. jejuni growth may have resulted from the adhesion of mucin oligosaccharide ligands to the bacterium (Hugdahl et al., 1988), creating a physical barrier that limited its capability to obtain nutrients. Another explanation could be that MUC2 itself has antimicrobial properties, as several mucin oligosaccharides have these properties. For example, the O-linked

Table 2. Net growth of C. jejuni NCTC 11168 in medium containing human mucin MUC2 at various concentrations after $12 \mathrm{~h}$ microaerobic incubation at $37{ }^{\circ} \mathrm{C}$

The data are given as the difference in $\log _{10}$ (c.f.u. $\mathrm{ml}^{-1}$ ) between 0 and $12 \mathrm{~h}$ at each MUC2 concentration. The controls were the cultures grown without MUC2. The data are given as means \pm SE from triplicates of three independent experiments.

\begin{tabular}{|lc|}
\hline MUC2 concn $\left(\mathbf{m g ~ m l}^{-1}\right)$ & $\boldsymbol{\Delta l o g}_{\mathbf{1 0}}\left(\right.$ c.f.u. $\left.\mathbf{~ m l}^{-1}\right)$ \\
\hline 0.00 & $2.7 \pm 0.2$ \\
0.01 & $2.5 \pm 0.1$ \\
0.05 & $1.7 \pm 0.1$ \\
0.20 & $1.6 \pm 0.1$ \\
2.00 & $1.5 \pm 0.1$ \\
\hline
\end{tabular}

oligosaccharides of the human gastric mucin MUC6 contain an $\alpha 1$,4-linked $\mathrm{N}$-acetylglucosamine able to inhibit the biosynthesis of cell-wall components of Helicobacter pylori (Kawakubo et al., 2004). Also, the 20-mer of MUC7 mucin has direct antifungal properties against Candida albicans via a histatin domain at its N-terminus (Bobek \& Situ, 2003). It is unlikely that increased viscosity was responsible for the altered growth, as reduced individual MUC2 subunits were used in these experiments. The rheological properties of mucus are attributable mainly to the polymerization of secreted mucins. In vivo, MUC2 is homo-oligomerized into complexes polymerized via disulfide bonds. The mucin aggregates give mucus its viscous properties and help retain many molecules, including antimicrobial ones, secreted by the mucosa. This study used low concentrations of MUC2 with reduced disulfide bonds and complexes disassembled into individual subunits. Reduction solubilizes the mucin and only at high concentrations $\left(>10 \mathrm{mg} \mathrm{ml}^{-1}\right.$ ) do suspensions become more viscous. Thus the mucin concentrations used in these experiments did not change the viscosity to any significant extent. This study did not attempt to duplicate the environment of mucus but rather to investigate the response of the bacteria to MUC2, the major constituent of human intestinal mucus. A $0.05 \mathrm{mg} \mathrm{ml}^{-1}$ concentration of MUC2 produced measurable inhibition of $C$. jejuni growth and was chosen to compare the characteristics of bacterial growth in the presence and absence of this mucin, and to investigate the transcriptomic regulation induced by its presence.

\section{Bacterial growth characteristics}

The expression of bacterial transcripts depends on the growth phase of the organism, as changes in gene regulation occur from the exponential to the stationary phases of growth. The time course of the growth of specific bacteria depends on strains, culture medium and incubation conditions such as atmosphere and temperature. $C$. jejuni strain NCTC 11168 growth curves in the presence or absence of MUC2 were constructed using the same experimental parameters as for the transcriptomics studies. Growth was measured at eight time points by counting the c.f.u. $\mathrm{ml}^{-1}$ of bacteria grown in liquid cultures. The growth of C. jejuni increased exponentially up to $15 \mathrm{~h}$ when it reached a maximum density of viable cells; after this time, a decline in the number of viable cells indicated the beginning of the stationary phase (Fig. 1). These results are in good agreement with previous data (Sampathkumar et al., 2006). There were no differences in the duration of the exponential growth phase between bacteria grown with or without MUC2 at this concentration.

\section{Regulation of expression of 20 C. jejuni genes encoding several functions}

The response of $C$. jejuni gene transcription to the presence of MUC2 was investigated in nine test cultures with 


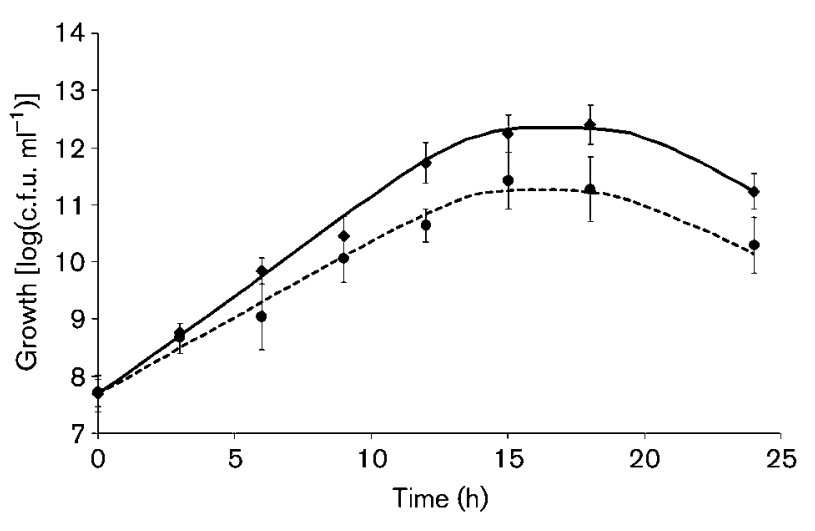

Fig. 1. Time course of C. jejuni NCTC 11168 grown in $\mathrm{BHI}$ medium with or without $0.05 \mathrm{mg} \mathrm{MUC2} \mathrm{ml}^{-1}$. Bacteria were grown under microaerobic conditions at $37{ }^{\circ} \mathrm{C}$. At $0,3,6,9,12,15,18$ and $24 \mathrm{~h}$, culture aliquots were taken to determine the number of c.f.u. $\mathrm{ml}^{-1}$ by inoculating different dilutions of the bacterial suspension on CSA plates and incubating them under microaerobic conditions for $24 \mathrm{~h}$. Bacterial growth in the absence of MUC2; $\bullet$, bacterial growth in the presence of $0.05 \mathrm{mg} \mathrm{MUC2}$ $\mathrm{ml}^{-1}$. The results are shown as means \pm SE from duplicates of two independent cultures.

$0.05 \mathrm{mg}$ MUC2 $\mathrm{ml}^{-1}$ and nine control cultures without mucin grown for $12 \mathrm{~h}$. The RNA was extracted and pooled into three biological replicates of each condition $(n=3)$. The six RNA samples were reverse-transcribed and each cDNA sample was amplified in three technical replicates. A total of 20 genes were selected for study in the functional categories encoding virulence factors and toxins, putative mucin-degrading enzymes, cell morphology and adhesion, and motility. Fifteen genes were significantly upregulated, including those encoding cytolethal distending toxin $(c d t A B C)$, vacuolating cytotoxin $(v a c B), C$. jejuni lipoprotein (jlpA), Campylobacter invasion antigen (ciaB), the multidrug efflux system ( $c m e A B)$, putative mucin-degrading enzymes (cj1344c, cj0843c, cj0256 and cj1055c), flagellin A $(f l a A)$ and putative rod-shape-determining proteins (mreBC). Expression of the genes encoding the Campylobacter adhesion fibronectin-binding protein $(c a d F)$ and sialic acid synthase (neuB1) were downregulated. Three genes encoding UTP-glucose-1-phosphate uridylyltransferase ( $g a l U)$, bacterioferritin $(b f r)$ and flagellin B $(f l a B)$ did not show significant modulation of expression (Fig. 2).

C. jejuni has several features to help it move through the highly viscous mucus gel: a cellular spiral shape, the ability to open up paths in the mucus layer and polar flagella (Ferrero \& Lee, 1988). In the presence of MUC2, upregulation was observed in the relative expression of $C$. jejuni genes encoding proteins with a role in cell-shape maintenance $(m r e B C)$, mucin-degrading enzymes ( $c j 1344 c$, $c j 0843 c, c j 0256$ and $c j 1055 c$ ) and motility (flaA). The MreB (mecillinam-resistance protein $B$ ) protein and its homo- logues can polymerize into linear protofilaments forming 'cables' that run around the cell (Egelman, 2003) and associate with membrane proteins such as $\mathrm{MreC}$, which serves to anchor it to the cell wall (Egelman, 2003). These proteins also play a role in bacterial adherence to host cells.

Degradation of mucin carbohydrates exposes the protein core to proteases, resulting in disruption of the macromolecular complexes and, consequently, the function of mucins in the mucus. For example, Vibrio cholerae HapA, which has both mucinolytic and cytotoxic activities, is induced by mucin and is required by the bacterium to traverse through mucin-containing gels (Silva et al., 2003). Several putative mucinolytic enzymes have been identified in the genome of C. jejuni strain NCTC 11168: the sialoglycoprotease $\mathrm{Cj} 1344 \mathrm{c}$, the murein lytic glycosylase $\mathrm{Cj0843c}$ and the sulfoglycolases Cj0256 and Cj1055c. Consistent with their functions, these four genes were upregulated by MUC2.

A determinant of host colonization by $C$. jejuni is the motility of the bacterium. A major component of its flagellar apparatus is the flagellin encoded by fla $A$ and a minor component is the flagellin encoded by flaB (Sommerlad \& Hendrixson, 2007). The transcription of both flagellins is under the regulation of different sigma factors, $\sigma^{28}$ for flaA and $\sigma^{54}$ for flaB (Hendrixson et al., 2001). Fully motile, wild-type $C$. jejuni bacteria express flaA, but expression of flaB is not required for motility (Wassenaar et al., 1993). MUC2 upregulated the expression of flaA but did not change that of flaB, suggesting that mucin serves as an environmental signal to enhance motility.

Adhesion to host cells is necessary to allow C. jejuni to remain in the intestine and invade the epithelium. Adherence involves different types of lectin-like molecules attaching to their corresponding glycosylated receptors on the epithelial cells (Guerry et al., 2002). Major outermembrane proteins such as the putative rod-shapedetermining protein $\mathrm{MreB}$, the putative periplasmic protein MreC and C. jejuni lipoprotein JlpA play important roles in bacterial adhesion (Ashgar et al., 2007; del Rocio Leon-Kempis et al., 2006; Jin et al., 2003; Kakuda \& DiRita, 2006; Karlyshev et al., 2000; Konkel et al., 1997; Linton et al., 2000; Mamelli et al., 2006; McSweegan \& Walker, 1986; Monteville \& Konkel, 2002; Moser et al., 1997; Pei \& Blaser, 1993). The genes encoding these three proteins were upregulated in response to MUC2.

Different factors and determinants may be expressed at various stages of infection to help the adaptation of a bacterium to different conditions. Some virulence factors help bacterial pathogens to attach themselves to cell surfaces, to invade particular host cells and potentially to modify cells or tissues to their advantage, and they can cause collateral damage at the same time (Chen et al., 2005). The gene ciaB encoding the Campylobacter invasion antigen $\mathrm{CiaB}$ was upregulated by MUC2; CiaB plays an important role in internalization of $C$. jejuni into 


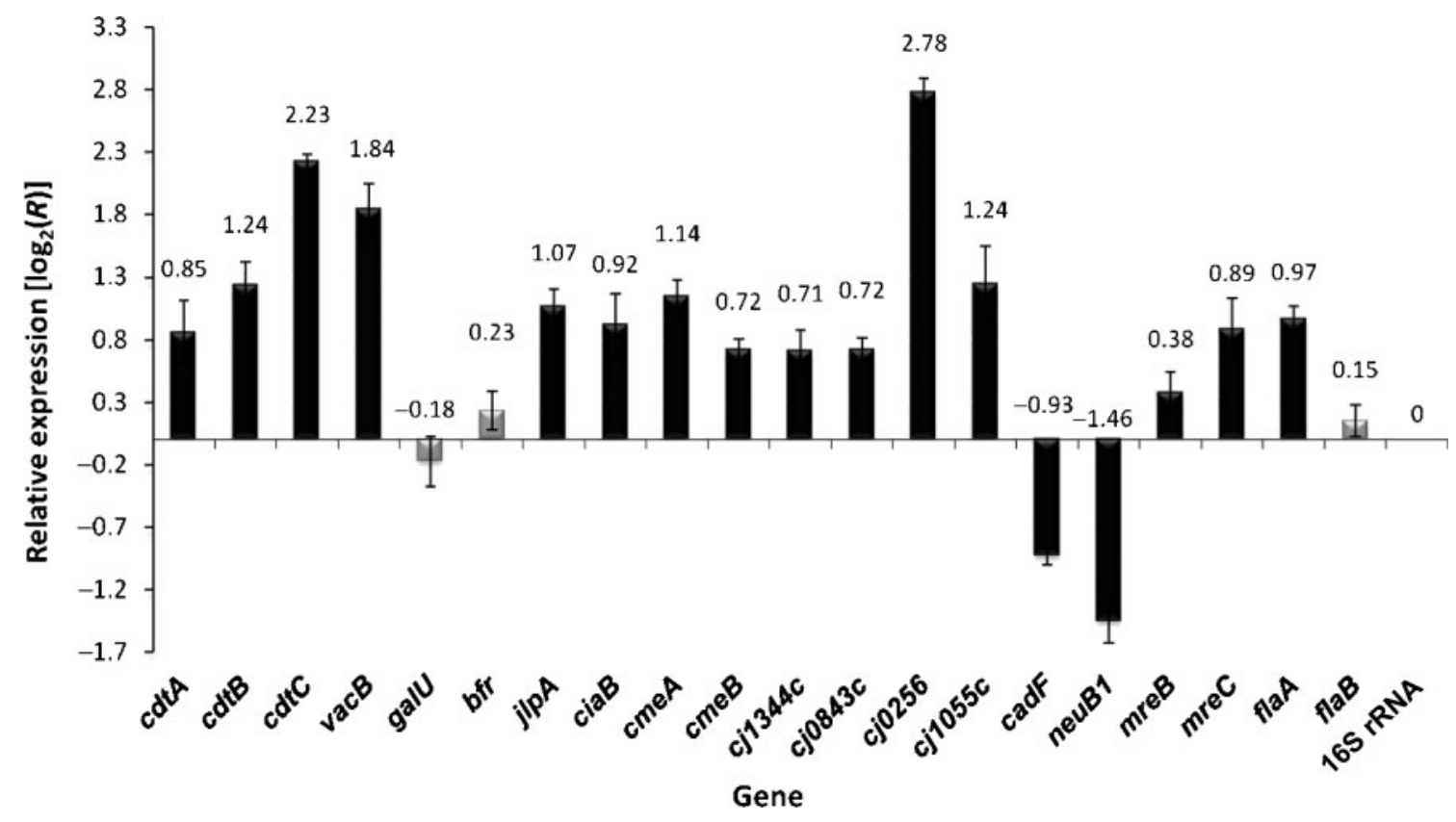

Fig. 2. Relative expression of 20 genes of $C$. jejuni NCTC 11168 after $12 \mathrm{~h}$ microaerobic incubation at $37{ }^{\circ} \mathrm{C}$ with $0.05 \mathrm{mg}$ human intestinal mucin MUC2 $\mathrm{ml}^{-1}$. Relative expression greater than $>0$ indicates upregulation and $<0$ indicates downregulation. Relative expression of a gene equal to \pm 1.0 indicates $100 \%$ modulation of expression in the presence of MUC2. The relative expression value is given above the histogram for each gene.

mammalian cells (Konkel et al., 1999). The genes neuB1 and $c a d F$ were downregulated by MUC2. NeuB1 is the main component of $\mathrm{N}$-acetylneuraminic acid synthetase involved in sialylation of lipo-oligosaccharides; $C$. jejuni lipo-oligosaccharides mimic human gangliosides and may serve to disguise the bacterium following bacterial infection (Linton et al., 2000). The gene cadF encodes a protein that helps C. jejuni to bind to host fibronectin (KrauseGruszczynska et al., 2007). NeuB1 and CadF are important for immune shielding and adhesion to the extracellular matrix, respectively. Thus it can be hypothesized that these proteins are not required to penetrate the mucus barrier, but their expression may be upregulated when the bacterium enters the epithelial microenvironment.

Toxins are considered major C. jejuni virulence factors contributing to cellular or tissue damage and disease induction. Cytolethal distending toxin (Cdt) is a secreted holotoxin present in many non-enteric and enteric pathogens (Lara-Tejero \& Galan, 2001; Lee et al., 2003). It comprises three subunits, $\mathrm{CdtA}, \mathrm{CdtB}$ and $\mathrm{CdtC}$, which act together to block cell division by performing cell-cycle arrest (Lara-Tejero \& Galan, 2001): CdtA and CdtC bind to the cell surface helping to deliver the active subunit CdtB, which uses its DNase I-like activity to cleave dsDNA molecules during the $G_{1}$ or $G_{2}$ phase (Whitehouse et al., 1998). The gene cluster $c d t A B C$ and the gene $v a c B$ encoding a vacuolating toxin were significantly upregulated by MUC2.
The genes encoding the multidrug efflux system $\mathrm{CmeAB}$ also were upregulated by MUC2. This efflux system is involved in resistance of $C$. jejuni against bile and other antimicrobial agents (Lin et al., 2002, 2005). Thus MUC2 may act as a cue to the bacterium to enhance its defences against host antimicrobial factors.

For the first time, we have shown here that $C$. jejuni has evolved an ability to respond to the presence of the human MUC2 intestinal mucin by modulation of expression of genes known to be important for colonization and pathogenicity (Fig. 2). Transcriptional activation of genes encoding cellmorphology proteins, motility proteins, adhesins and virulence factors upon entering intestinal mucus would facilitate C. jejuni colonization of mucus and attachment to and invasion of the underlying mucosal epithelial cells.

The response of $C$. jejuni to MUC2 may not be specific to MUC2 or even to human mucins. It is likely to be mediated through oligosaccharide structures that could also be present on intestinal cell-surface mucins, secreted mucins from other regions of the gastrointestinal tract and other mucosal tissues, or intestinal mucins from other mammalian and avian species (Karlsson et al., 1997; Robbe et al., 2004; Schulz et al., 2002). However, MUC2 is virtually the sole secreted human intestinal mucin, and this work aimed to understand changes in the transcriptome of $C$. jejuni when it infects the human intestine and interacts with a mucin found there. MUC6 is secreted in the stomach but not in the intestine, and its concentration in 
intestinal mucus is bound to be very small. Shed extracellular domains of intestinal cell-surface mucins such as MUC12, -13 and -17 can be found within mucus at low concentrations. Small amounts of these mucins could have been present in our preparations, but PAS staining of blots showed only a single mucin band that coincided with MUC2, indicating that any contamination with other mucins was at a very low level.

The observation that $C$. jejuni is responsive to MUC2 raises the question of the identity and nature of the bacterial MUC2 receptor, which could possibly be a lectin-binding oligosaccharide. Oligosaccharides vary throughout the gastrointestinal tract, among different mucosal tissues and among species. Moreover, because of inherited differences in the glycosyltransferases that build these structures, interindividual differences in mucin glycosylation within individual mucosal tissues occur within human populations. In the case of gastric mucins and the related bacterium $H$. pylori, these host differences influence their susceptibility to various strains of the bacterium (Aspholm-Hurtig et al., 2004; Lindén et al., 2008). In future work, it will be of interest to investigate which intestinal mucin oligosaccharides trigger $C$. jejuni responses, and whether differences in the expression of these structures among individuals and species underlie differential susceptibilities to pathology following infection. If this were the case, the different glycosylation of MUC2 in various mammalian and avian species could modulate the expression of different $C$. jejuni genes in the intestinal microenvironments of those hosts and explain the differential pathogenicity of $C$. jejuni to them. Identification of the bacterial receptors could thus provide new molecular therapeutic targets against $C$. jejuni infection.

\section{ACKNOWLEDGEMENTS}

The support of NHMRC Project Grant 382309 and a UNSW Faculty of Medicine Grant are gratefully acknowledged. M.A.McG. was supported by a Queensland Cancer Council Senior Research Fellowship.

\section{REFERENCES}

Allos, B. M. (2001). Campylobacter jejuni infections: update on emerging issues and trends. Clin Infect Dis 32, 1201-1206.

Altschul, S. F., Madden, T. L., Schaffer, A. A., Zhang, J., Miller, W. \& Lipman, D. J. (1997). Gapped BLAST and PSI-BLAST: a new generation of protein database search programs. Nucleic Acids Res 25, 3389-3402.

Ashgar, S. S. A., Oldfield, N. J., Wooldridge, K. G., Jones, M. A., Irving, G. J., Turner, D. P. J. \& Ala'Aldeen, D. A. A. (2007). CapA, an autotransporter protein of Campylobacter jejuni, mediates association with human epithelial cells and colonization of the chicken gut. $J$ Bacteriol 189, 1856-1865.

Aspholm-Hurtig, M., Dailide, G., Lahmann, M., Kalia, A., Ilver, D. Roche, N., Vikström, S., Sjöström, R., Lindén, S. \& other authors (2004). Functional adaptation of BabA, the H. pylori ABO blood group antigen binding adhesin. Science 305, 519-522.

Bobek, L. A. \& Situ, H. (2003). MUC7 20-mer: investigation of antimicrobial activity, secondary structure, and possible mechanism of antifungal action. Antimicrob Agents Chemother 47, 643-652.
Chen, L., Yang, J., Yu, J., Yao, Z., Sun, L., Shen, Y. \& Jin, O. (2005). VFDB: a reference database for bacterial virulence factors. Nucleic Acids Res 33, D325-D328.

Davies, J. R. \& Carlstedt, I. (2000). Isolation of large gel-forming mucins. Methods Mol Biol 125, 3-13.

del Rocio Leon-Kempis, M., Guccione, E., Mulholland, F., Williamson, M. P. \& Kelly, D. J. (2006). The Campylobacter jejuni $\mathrm{PEBla}$ adhesin is an aspartate/glutamate-binding protein of an $\mathrm{ABC}$ transporter essential for microaerobic growth on dicarboxylic amino acids. Mol Microbiol 60, 1262-1275.

Egelman, E. H. (2003). Actin's prokaryotic homologs. Curr Opin Struct Biol 13, 244-248.

Ferrero, R. L. \& Lee, A. (1988). Motility of Campylobacter jejuni in a viscous environment: comparison with conventional rod-shaped bacteria. J Gen Microbiol 134, 53-59.

Gerharz, E. W., Turner, W. H., Kälble, T. \& Woodhouse, C. R. (2003). Metabolic and functional consequences of urinary tract reconstruction with bowel. BJU Int 91, 143-149.

Guerry, P., Szymanski, C. M., Prendergast, M. M., Hickey, T. E., Ewing, C. P., Pattarini, D. L. \& Moran, A. P. (2002). Phase variation of Campylobacter jejuni 81-176 lipooligosaccharide affects ganglioside mimicry and invasiveness in vitro. Infect Immun 70, 787-793.

Hendrixson, D. R., Akerley, B. J. \& DiRita, V. J. (2001). Transposon mutagenesis of Campylobacter jejuni identifies a bipartite energy taxis system required for motility. Mol Microbiol 40, 214-224.

Hugdahl, M. B., Beery, J. T. \& Doyle, M. P. (1988). Chemotactic behavior of Campylobacter jejuni. Infect Immun 56, 1560-1566.

Jin, S., Song, Y. C., Emili, A., Sherman, P. M. \& Chan, V. L. (2003). JlpA of Campylobacter jejuni interacts with surface-exposed heat shock protein $90 \alpha$ and triggers signalling pathways leading to the activation of NF- $\kappa \mathrm{B}$ and p38 MAP kinase in epithelial cells. Cell Microbiol 5, 165-174.

Kakuda, T. \& DiRita, V. J. (2006). Cj1496c encodes a Campylobacter jejuni glycoprotein that influences invasion of human epithelial cells and colonization of the chick gastrointestinal tract. Infect Immun 74, 4715-4723.

Karlsson, N. G., Herrmann, A., Karlsson, H., Johansson, M. E. V., Carlstedt, I. \& Hansson, G. C. (1997). The glycosylation of rat intestinal Muc2 mucin varies between rat strains and the small and large intestine. A study of O-linked oligosaccharides by a mass spectrometric approach. J Biol Chem 272, 27025-27034.

Karlyshev, A. V., Linton, D., Gregson, N. A., Lastovica, A. J. \& Wren, B. W. (2000). Genetic and biochemical evidence of a Campylobacter jejuni capsular polysaccharide that accounts for Penner serotype specificity. Mol Microbiol 35, 529-541.

Kawakubo, M., Ito, Y., Okimura, Y., Kobayashi, M., Sakura, K., Kasama, S., Fukuda, M. N., Fukuda, M., Katsuyama, T. \& Nakayama, J. (2004). Natural antibiotic function of a human gastric mucin against Helicobacter pylori infection. Science 305, 1003-1006.

Konkel, M. E., Garvis, S. G., Tipton, S. L., Anderson, D. E., Jr \& Cieplak, W., Jr (1997). Identification and molecular cloning of a gene encoding a fibronectin-binding protein (CadF) from Campylobacter jejuni. Mol Microbiol 24, 953-963.

Konkel, M. E., Kim, B. J., Rivera-Amill, V. \& Garvis, S. G. (1999). Bacterial secreted proteins are required for the internalization of Campylobacter jejuni into cultured mammalian cells. Mol Microbiol 32, 691-701.

Krause-Gruszczynska, M., van Alphen, L. B., Oyarzabal, O. A., Alter, T., Hänel, I., Schliephake, A., König, W., van Putten, J. P., Konkel, M. E. \& Backert, S. (2007). Expression patterns and role of the CadF protein in Campylobacter jejuni and Campylobacter coli. FEMS Microbiol Lett 274, 9-16. 
Lara-Tejero, M. \& Galan, J. E. (2001). CdtA, CdtB, and CdtC form a tripartite complex that is required for cytolethal distending toxin activity. Infect Immun 69, 4358-4365.

Lecuit, M., Abachin, E., Martin, A., Poyart, C., Pochart, P., Suarez, F., Bengoufa, D., Feuillard, J., Lavergne, A. \& other authors (2004). Immunoproliferative small intestinal disease associated with Campylobacter jejuni. N Engl J Med 350, 239-248.

Lee, R. B., Hassane, D. C., Cottle, D. L. \& Pickett, C. L. (2003). Interactions of Campylobacter jejuni cytolethal distending toxin subunits CdtA and CdtC with HeLa cells. Infect Immun 71, 48834890.

Lin, J., Michel, L. O. \& Zhang, Q. (2002). CmeABC functions as a multidrug efflux system in Campylobacter jejuni. Antimicrob Agents Chemother 46, 2124-2131.

Lin, J., Cagliero, C., Guo, B., Barton, Y.-W., Maurel, M.-C., Payot, S. \& Zhang, Q. (2005). Bile salts modulate expression of the CmeABC multidrug efflux pump in Campylobacter jejuni. J Bacteriol 187, 74177424.

Lindén, S., Mahdavi, J., Semino-Mora, C., Olsen, C., Carlstedt, I., Boren, T. \& Dubois, A. (2008). Role of ABO secretor status in mucosal innate immunity and H. pylori infection. PLoS Pathog 4, e2.

Linton, D., Karlyshev, A. V., Hitchen, P. G., Morris, H. R., Dell, A., Gregson, N. A. \& Wren, B. W. (2000). Multiple $N$-acetyl neuraminic acid synthetase $(n e u B)$ genes in Campylobacter jejuni: identification and characterization of the gene involved in sialylation of lipooligosaccharide. Mol Microbiol 35, 1120-1134.

Livak, K. J. \& Schmittgen, T. D. (2001). Analysis of relative gene expression data using real-time quantitative PCR and the $2^{-\Delta \Delta C}$ method. Methods 25, 402-408.

Mamelli, L., Pages, J.-M., Konkel, M. E. \& Bolla, J.-M. (2006). Expression and purification of native and truncated forms of CadF, an outer membrane protein of Campylobacter. Int J Biol Macromol 39, $135-140$.

McAuley, J. L., Linden, S. K., Png, C. W., King, R. M., Pennington, H. L., Gendler, S. J., Florin, T. H., Hill, G. R., Korolik, V. \& McGuckin, M. A. (2007). MUC1 cell surface mucin is a critical element of the mucosal barrier to infection. J Clin Invest 117, 2313-2324.

McSweegan, E. \& Walker, R. I. (1986). Identification and characterization of two Campylobacter jejuni adhesins for cellular and mucous substrates. Infect Immun 53, 141-148.

Monteville, M. R. \& Konkel, M. E. (2002). Fibronectin-facilitated invasion of T84 eukaryotic cells by Campylobacter jejuni occurs preferentially at the basolateral cell surface. Infect Immun 70, 66656671.

Moser, I., Schroeder, W. \& Salnikow, J. (1997). Campylobacter jejuni major outer membrane protein and a 59-kDa protein are involved in binding to fibronectin and INT 407 cell membranes. FEMS Microbiol Lett 157, 233-238.

Nabi, G., N'Dow, J., Hasan, T. S., Booth, I. R. \& Cash, P. (2005). Proteomic analysis of urine in patients with intestinal segments transposed into the urinary tract. Proteomics 5, 1729-1733.
Neutra, M. R. \& Forstner, J. F. (1987). Gastrointestinal mucus: synthesis, secretion, and function. In Physiology of the Gastrointestinal Tract, pp. 975-1009. Edited by L. R. Jonson. New York: Raven Press.

Parkhill, J., Wren, B. W., Mungall, K., Ketley, J. M., Churcher, C., Basham, D., Chillingworth, T., Davies, R. M., Feltwell, T. \& other authors (2000). The genome sequence of the food-borne pathogen Campylobacter jejuni reveals hypervariable sequences. Nature 403, 665-668.

Pei, Z. \& Blaser, M. J. (1993). PEB1, the major cell-binding factor of Campylobacter jejuni, is a homolog of the binding component in Gram-negative nutrient transport systems. J Biol Chem 268, 1871718725.

Pfaffl, M. W., Horgan, G. W. \& Dempfle, L. (2002). Relative expression software tool (RESTC) for group-wise comparison and statistical analysis of relative expression results in real-time PCR. Nucleic Acids Res 30, e36.

Robbe, C., Capon, C., Coddeville, B. \& Michalski, J.-C. (2004). Structural diversity and specific distribution of $O$-glycans in normal human mucins along the intestinal tract. Biochem J 384, 307-316.

Rozen, S. \& Skaletsky, H. J. (1998). Primer3. Code available at http:// primer3.sourceforge.net/ and BioManager by ANGIS (http://www. angis.org.au).

Sampathkumar, B., Napper, S., Carrillo, C. D., Willson, P., Taboada, E., Nash, J. H. E., Potter, A. A., Babiuk, L. A. \& Allan, B. J. (2006). Transcriptional and translational expression patterns associated with immobilized growth of Campylobacter jejuni. Microbiology 152, 567577.

Schulz, B. L., Packer, N. H. \& Karlsson, N. G. (2002). Small-scale analysis of $\mathrm{O}$-linked oligosaccharides from glycoproteins and mucins separated by gel electrophoresis. Anal Chem 74, 6088-6097.

Silva, A. J., Pham, K. \& Benitez, J. A. (2003). Haemagglutinin/protease expression and mucin gel penetration in El Tor biotype Vibrio cholerae. Microbiology 149, 1883-1891.

Sommerlad, S. M. \& Hendrixson, D. R. (2007). Analysis of the roles of FlgP and FlgQ in flagellar motility of Campylobacter jejuni. J Bacteriol 189, 179-186.

Strugala, V., Allen, A., Dettmar Peter, W. \& Pearson Jeffrey, P. (2003). Colonic mucin: methods of measuring mucus thickness. Proc Nutr Soc 62, 237-243.

Szymanski, C. M., King, M., Haardt, M. \& Armstrong, G. D. (1995). Campylobacter jejuni motility and invasion of Caco-2 cells. Infect Immun 63, 4295-4300.

Wassenaar, T. M., van der Zeijst, B. A., Ayling, R. \& Newell, D. G. (1993). Colonisation of chicks by motility mutants of C. jejuni demonstrates the importance of flagellin A expression. J Gen Microbiol 139, 1171-1175.

Whitehouse, C. A., Balbo, P. B., Pesci, E. C., Cottle, D. L., Mirabito, P. M. \& Pickett, C. L. (1998). Campylobacter jejuni cytolethal distending toxin causes a $\mathrm{G}_{2}$-phase cell cycle block. Infect Immun 66, 1934-1940.

Yuki, N. \& Koga, M. (2006). Bacterial infections in Guillain-Barré and Fisher syndromes. Curr Opin Neurol 19, 451-457. 